\title{
ON AN INVERSION FORMULA FOR THE LAPLACE TRANSFORMATION
}

\author{
P. G. ROONEY
}

1. Introduction. The literature of the Laplace transformation contains many examples of inversion operators. Particular attention has been given in this literature to the so-called "real" inversion operators, that is, those operators which make use of values of the generating function arising only from real values of the independent variable.

It is one of these "real" inversion operators which we shall study here, one which has already been studied in part. If

$$
f(s)=\int_{0}^{\infty} e^{-s t} \phi(t) d t
$$

then the inversion operator is defined by the formula

$$
{ }^{\nu} L_{k, t}[f(s)]=\frac{k^{3 / 2} e^{2 k}}{t \pi^{\frac{1}{2}}} \int_{0}^{\infty} x^{\frac{1}{2} \nu} J_{\nu}\left(2 k x^{\frac{1}{2}}\right) f\left(\frac{k(x+1)}{t}\right) d x
$$

and we shall show that, under certain conditions,

$$
\lim _{k \rightarrow \infty}{ }^{\nu} L_{k, t}[f(s)]=\phi(t) .
$$

The operator was originally given by Erdélyi (1). However the resulting inversion and representation theories were not studied there. A special case of the operator, namely $\nu=-\frac{1}{2}$, was studied by the author in (2).

We shall derive our results here partially from those of (2) by means of a relation, also derived here, between the operators arising from different values of $\nu$.

In $\$ 2$ we find conditions for the existence of the operator, and derive a number of its properties, including the one mentioned in the previous paragraph.

Section 3 contains the inversion theory, and in $\$ 4$ we find necessary and sufficient conditions that a function be equal almost everywhere to a Laplace transform of a function of the form $t^{\alpha} \phi(t), \phi \in L_{p}(0, \infty), 1<p \leqslant \infty$, $\alpha>-(p-1) / p$.

The notations introduced by formulas I and II will be taken as standard throughout this paper, as well as

III

$$
\begin{aligned}
f_{\mu}(s) & =\int_{0}^{\infty} e^{-s t} t^{-\mu} \phi(t) d t \\
\bar{f}(s) & =\int_{0}^{\infty} e^{-s t}|\phi(t)| d t .
\end{aligned}
$$

Received December 11, 1953. 
2. Existence and properties of the operator. The following theorem gives sufficient conditions for the operator to exist for a given $\phi(t)$.

THEOREM 2.1. If

$$
\begin{aligned}
e^{-\gamma t} \phi(t) & \in L(0, \infty), \quad \gamma>0, \\
t^{-\left(\frac{1}{2} \nu+3 / 4\right)} \phi(t) & \in L(0, \delta), \text { for some } \delta>0, \\
& \nu>-1,
\end{aligned}
$$

then for each $t>0$, and all $k>\gamma t,{ }^{\nu} L_{k, t}[\bar{f}(s)]$ and ${ }^{\nu} L_{k, t}[f(s)]$ exist.

Proof. Clearly the existence of ${ }^{\nu} L_{k, t}[\bar{f}(s)]$ implies that of ${ }^{\nu} L_{k, t}[f(s)]$, since $|f(s)| \leqslant \bar{f}(s)$. Let $k>\gamma t$, and $t>0$. Now by $(3 ; \S 7.21)$

$$
J_{\nu}(x) \sim\left(\frac{2}{\pi x}\right)^{\frac{1}{2}} \cos \left(x-\frac{1}{2} \nu \pi-\frac{1}{4} \pi\right) \text { as } x \rightarrow \infty,
$$

and hence $R>0$ and $M$ exist such that $\left|J_{\nu}(x)\right| \leqslant M x^{-\frac{1}{2}}$ for $x \geqslant R$. Thus

$$
\begin{aligned}
& \int_{R / 2 k}^{\infty} e^{-k u y^{2} / t} y^{\nu+1}\left|J_{\nu}(2 k y)\right| d y \\
& \quad \leqslant(2 k)^{-\frac{1}{2}} M \int_{0}^{\infty} e^{-k u y^{2} / t} y^{\nu+\frac{1}{2}} d y=(2 / k)^{\frac{1}{2}} M \Gamma\left(\frac{1}{2} \nu+\frac{3}{4}\right)(k u / t)^{-\left(\frac{1}{2} \nu+3 / 4\right)} .
\end{aligned}
$$

Further, since $\nu>-1$, the integral,

$$
\int_{0}^{R / 2 k} e^{-k u y^{2} / t} y^{\nu+1}\left|J_{\nu}(2 k y)\right| d y
$$

is uniformly bounded in $u$ for $u \geqslant 0$. Hence,

$$
\begin{aligned}
& \int_{0}^{\infty} e^{-k u / t}|\phi(u)| d u \int_{0}^{\infty} e^{-k u y^{2} / t} y^{\nu+1}\left|J_{\nu}(2 k y)\right| d y \\
& \quad=\int_{0}^{\infty} e^{-k u / t}|\phi(u)| d u\left\{\int_{0}^{R / 2 k}+\int_{R / 2 k}^{\infty}\right\} e^{-k u y^{2} / t} y^{\nu+1}\left|J_{\nu}(2 k y)\right| d y \\
& \leqslant M_{1} \int_{0}^{\infty} e^{-k u / t}|\phi(u)| d u+M_{2} \int_{0}^{\infty} e^{-k u / t} u^{-\left(\frac{1}{2} \nu+3 / 4\right)}|\phi(u)| d u<\infty
\end{aligned}
$$

by (1) and (2), and where

$$
M_{1}=\int_{0}^{R / 2 k} y^{\nu+1}\left|J_{\nu}(2 k y)\right| d y \text {, and } M_{2}=M \Gamma\left(\frac{1}{2} \nu+\frac{3}{4}\right)(2 / k)^{\frac{1}{2}}(k u / t)^{-\left(\frac{1}{2} \nu+3 / 4\right)} .
$$

Hence, by Fubini's theorem we have that

$$
\begin{aligned}
& \frac{2 k^{3 / 2} e^{2 k}}{t \pi^{\frac{1}{2}}} \int_{0}^{\infty} e^{-k u / t}|\phi(u)| d u \int_{0}^{\infty} e^{-k u y^{2} / t} y^{\nu+1} J_{\nu}(2 k y) d y \\
& \quad=\frac{2 k^{3 / 2} e^{2 k}}{t \pi^{\frac{1}{2}}} \int_{0}^{\infty} y^{\nu+1} J_{\nu}(2 k y) d y \int_{0}^{\infty} e^{-k\left(y^{2}+1\right) u / t}|\phi(u)| d u \\
& \left.\quad=\frac{k^{3 / 2} e^{2 k}}{t \pi^{\frac{1}{2}}} \int_{0}^{\infty} x^{\frac{1}{2} \nu} J_{\nu}\left(2 k x^{\frac{1}{2}}\right) \int_{0}^{\infty} e^{-k(x+1) u / t}|\phi(u)| d u \quad \quad \text { (where } x=y^{2}\right) \\
& =\frac{k^{3 / 2} e^{2 k}}{t \pi^{\frac{1}{2}}} \int_{0}^{\infty} x^{\frac{1}{2} \nu} J_{\nu}\left(2 k x^{\frac{1}{2}}\right) \bar{f}\left(\frac{k(x+1)}{t}\right) d x={ }^{\nu} L_{k, t}[\bar{f}(s)]
\end{aligned}
$$

exists for each $t>0$ and all $k>\gamma t$. 
Corollary. If

$$
\begin{aligned}
e^{-\gamma t} \phi(t) & \in L(0, \infty), \gamma>0, \\
\nu & >-1,
\end{aligned}
$$

then for each $t>0$ and all $k>\gamma t$, and each $\epsilon>0,{ }^{\nu} L_{k, t}\left[e^{-\epsilon s} f(s)\right]$ exists.

Proof. $e^{-\epsilon s} f(s)=\int_{0}^{\infty} e^{-s t} \phi_{\epsilon}(t) d t$, where $\phi_{\epsilon}(t)=0$, for $0<t<\epsilon$, and $\phi_{\epsilon}(t)=$ $\phi(t-\epsilon)$ for $t>\epsilon$. Thus the conditions of Theorem 2.1 are clearly fulfilled relative to $\phi_{\epsilon}(t)$, and ${ }^{\nu} L_{k, t}\left[e^{-\epsilon s} f(s)\right]$ exists.

The next theorem relates the operators arising from different values of $\nu$.

THEOREM 2.2. If

$$
\begin{gathered}
e^{-\gamma^{t} \phi(t)} \in L(0, \infty), \quad \gamma>0, \\
t^{-\left(\frac{1}{2} \lambda+\mu+3 / 4\right)} \phi(t) \in L(0, \delta) \text { for some } \delta>0, \\
\lambda>-1, \quad \mu \geqslant 0,
\end{gathered}
$$

then $f_{\mu}(s)$ exists for $s>\gamma$,

$$
{ }^{\lambda+\mu} L_{k, t}[f(s)] \text { and }{ }^{\lambda} L_{k, t}\left[f_{\mu}(s)\right]
$$

exists for each $t>0$ and all $k>\gamma t$, and for all such $k$ and $t$,

$$
{ }^{\lambda+\mu} L_{k, t}[f(s)]=t^{\mu} \lambda L_{k, t}\left[f_{\mu}(s)\right] .
$$

Proof. The existence of $f_{\mu}(s)$ is clear. The existence of

$$
{ }^{\lambda+\mu} L_{k, t}[f(s)] \text { and of }{ }^{\lambda} L_{k, t}\left[f_{\mu}(s)\right]
$$

follows from Theorem 2.1 .

Now by $(3 ; \S 12.11)$, for $\lambda>-1, \mu>0$,

$$
J_{\lambda+\mu}(z)=\frac{z^{\mu}}{2^{\mu-1} \Gamma(\mu)} \int_{0}^{\frac{1}{2} \pi} J_{\lambda}(z \sin \theta) \sin ^{\lambda+1} \theta \cos ^{2 \mu-1} \theta d \theta .
$$

Hence, setting $z=2 k x^{\frac{1}{2}}$, and $z \sin \theta=2 k u^{\frac{1}{2}}$, we have

$$
x^{\frac{1}{3}(\lambda+\mu)} J_{\lambda+\mu}\left(2 k x^{\frac{1}{2}}\right)=\frac{k^{\mu}}{\Gamma(\mu)} \int_{0}^{x} u^{\frac{1}{2} \lambda} J_{\lambda}\left(2 k u^{\frac{1}{2}}\right)(x-u)^{\mu-1} d u .
$$

Further, for $\mu>0, u \geqslant 0$, and $k \geqslant \gamma t>0$,

$$
\begin{aligned}
\int_{u}^{\infty}(x-u)^{\mu-1} f\left(\frac{k(x+1)}{t}\right) d x=\int_{u}^{\infty}(x-u)^{\mu-1} d x \int_{0}^{\infty} e^{-k(x+1) y / t} \phi(y) d y \\
=\int_{0}^{\infty} e^{-k y / t} \phi(y) d y \int_{u}^{\infty} e^{-k x y / t}(x-u)^{\mu-1} d x \\
=\left(\frac{t}{k}\right)^{\mu} \Gamma(\mu) \int_{0}^{\infty} e^{-k(u+1) y / t} y^{-\mu} \phi(y) d y \\
=\left(\frac{t}{k}\right)^{\mu} \Gamma(\mu) f_{\mu}\left(\frac{k(u+1)}{t}\right),
\end{aligned}
$$

the interchange of integrations being valid by Fubini's theorem since

$$
\begin{aligned}
\int_{0}^{\infty} e^{-k y / t}|\phi(y)| d y \int_{u}^{\infty} e^{-k x y / t}(x-u)^{\mu-1} d u \\
=\left(\frac{t}{k}\right)^{\mu} \Gamma(\mu) \int_{0}^{\infty} e^{-k(u+1) y / t} y^{-\mu}|\phi(y)| d y<\infty .
\end{aligned}
$$


Thus for $k>\gamma t>0$,

$$
\begin{aligned}
{ }^{\lambda+\mu} L_{k, t}[f(s)] & =\frac{k^{3 / 2} e^{2 k}}{t \pi^{\frac{1}{2}}} \int_{0}^{\infty} x^{\frac{1}{2}(\lambda+\mu)} J_{\lambda+\mu}\left(2 k x^{\frac{1}{2}}\right) f\left(\frac{k(x+1)}{t}\right) d x \\
& =\frac{k^{\mu+3 / 2} e^{2 k}}{t \pi^{\frac{1}{2}} \Gamma(\mu)} \int_{0}^{\infty} f\left(\frac{k(x+1)}{t}\right) d x \int_{0}^{x} u^{\frac{1}{2} \lambda} J_{\lambda}\left(2 k u^{\frac{1}{2}}\right)(x-u)^{\mu-1} d u \\
& =\frac{k^{\mu+3 / 2} e^{2 k}}{t \pi^{\frac{1}{2}} \Gamma(\mu)} \int_{0}^{\infty} u^{\frac{1}{2} \lambda} J_{\lambda}\left(2 k u^{\frac{1}{2}}\right) d u \int_{u}^{\infty}(x-u)^{\mu-1} f\left(\frac{k(x+1)}{t}\right) d x \\
& =\frac{t^{\mu} k^{3 / 2} e^{2 k}}{t \pi^{\frac{3}{3}}} \int_{0}^{\infty} u^{\frac{1}{2} \lambda} J_{\lambda}\left(2 k u^{\frac{1}{2}}\right) f_{\mu}\left(\frac{k(u+1)}{t}\right) d u \\
& =t^{\mu} L_{k, t}\left[f_{\mu}(s)\right],
\end{aligned}
$$

the interchange of integrations being justified by Fubini's theorem, since by Theorem $2.1{ }^{\lambda} L_{k, t}\left[\bar{f}_{\mu}(s)\right]$ exists and thus

$$
\begin{aligned}
& \int_{0}^{\infty} u^{\frac{1}{2} \lambda}\left|J_{\lambda}\left(2 k u^{\frac{1}{2}}\right)\right| d u \int_{u}^{\infty}(x-u)^{\mu-1}\left|f\left(\frac{k(x+1)}{t}\right)\right| d x \\
\leqslant & \int_{0}^{\infty} u^{\frac{1}{2} \lambda}\left|J_{\lambda}\left(2 k u^{\frac{1}{2}}\right)\right| d u \int_{u}^{\infty}(x-u)^{\mu-1} \bar{f}\left(\frac{k(x+1)}{t}\right) d x \\
= & \left(\frac{t}{k}\right)^{\mu} \Gamma(\mu) \int_{0}^{\infty} u^{\frac{1}{2} \lambda}\left|J_{\lambda}\left(2 k u^{\frac{1}{2}}\right)\right| \bar{f}_{\mu}\left(\frac{k(u+1)}{t}\right) d u<\infty .
\end{aligned}
$$

The theorem is clearly true if $\mu=0$.

Corollary. If

$$
\begin{aligned}
& e^{-\gamma t} \phi(t) \in L(0, \infty), \quad \gamma>0, \\
& t^{-\left(\frac{1}{2} \lambda+3 / 4\right)} \phi(t) \in L(0, \delta), \text { for some } \delta>0, \\
& \lambda>-1, \mu \geqslant 0,
\end{aligned}
$$

then $f_{-\mu}(s)$ exists for each $s>\gamma$.

$$
{ }^{\lambda+\mu} L_{k, t}\left[f_{-\mu}(s)\right] \text { and }{ }^{\lambda} L_{k, t}[f(s)]
$$

exist for each $t>0$ and all $k>\gamma t$, and for all such $k$ and $t$,

$$
{ }^{\lambda+\mu} L_{k, t}\left[f_{-\mu}(s)\right]=t^{\mu}{ }^{\lambda} L_{k, t}[f(s)] .
$$

Proof. The existence of $f_{-\mu}(s)$ is clear. Let $\psi(t)=t^{\mu} \phi(t)$ and

$$
g(s)=\int_{0}^{\infty} e^{-s t} \psi(t) d t=f_{-\mu}(s) .
$$

Clearly

for any $\gamma_{1}>\gamma$, and

$$
e^{-\gamma_{1} t} \psi(t) \in L(0, \infty)
$$

$$
t^{-\left(\frac{1}{2} \lambda+\mu+3 / 4\right)} \psi(t)=t^{-\left(\frac{1}{2} \lambda+3 / 4\right)} \phi(t) \in L(0, \delta) .
$$

Hence ${ }^{\lambda+\mu} L_{k, t}[g(s)]$ and $t^{\mu} \lambda L_{k, t}\left[g_{\mu}(s)\right]$ exist and are equal, by Theorem 2.2, for each $t>0$ and all $k>\gamma_{1} t$, and hence since $\gamma_{1}$ was an arbitrary number larger than $\gamma$, for all $k>\gamma t$. But $g(s)=f_{-\mu}(s), g_{\mu}(s)=f(s)$, and thus the statement is proved. 
Let $I_{\alpha}\{\phi(u) ; t\}$ denote the Riemann-Liouville fractional integral of order $\alpha$, i.e.,

$$
I_{\alpha}\{\phi(u) ; t\}= \begin{cases}(\Gamma(\alpha))^{-1} \int_{0}^{t}(t-u)^{\alpha-1} \phi(u) d u, & \alpha>0, \\ \phi(t), & \alpha=0 .\end{cases}
$$

The next theorem relates the inversion operator and the fractional integral.

THEOREM 2.3. If

$$
\begin{aligned}
& e^{-\gamma t} \phi(t) \in L(0, \infty), \quad \gamma>0, \\
& t^{-\left(\frac{1}{2} \nu+3 / 4\right)} \phi(t) \in L(0, \delta), \text { for some } \delta>0, \\
& \nu>-1, \quad \alpha \geqslant 0,
\end{aligned}
$$

then for each $t>0$, and all $k>\gamma t$,

$$
{ }^{\nu+\alpha} L_{k, t}\left[s^{-\alpha} f(s)\right] \text { and } I_{\alpha}\left\{{ }^{p} L_{k, u}[f(s)] ; t\right\}
$$

exist and are equal.

Proof. $s^{-\alpha} f(s)$ is the Laplace transform of $I_{\alpha}\{\phi(u) ; t\}$, which transform exists, by $(4 ;$ ch. $2, \S 8)$, for $s \geqslant \gamma$. Hence, by Theorem 2.1 ,

$$
{ }^{\nu+\alpha} L_{k, t}\left[s^{-\alpha} f(s)\right]
$$

will exist, for each $t>0$ and all $k>\gamma t$, if $t^{-\left(\frac{1}{2}(\nu+\alpha)+3 / 4\right)} I_{\alpha}\{\phi(u) ; t\} \in L(0, \delta)$ for some $\delta>0$. But, using the same $\delta$ as in (2), we have

$$
\begin{aligned}
& \int_{0}^{\delta} t^{-\left(\frac{1}{2}(\nu+\alpha)+3 / 4\right)}\left|I_{\alpha}\{\phi(u) ; t\}\right| d t \\
& \quad \leqslant(\Gamma(\alpha))^{-1} \int_{0}^{\delta} t^{-\left(\frac{1}{2}(\nu+\alpha)+3 / 4\right)} d t \int_{0}^{t}(t-u)^{\alpha-1}|\phi(u)| d u \\
& \quad=(\Gamma(\alpha))^{-1} \int_{0}^{\delta} t^{-\left(\frac{1}{2}(\nu+\alpha)+3 / 4\right)} d t \int_{0}^{t}(t-u)^{\frac{1}{2} \alpha-1}(t-u)^{\frac{1}{2} \alpha}|\phi(u)| d u \\
& \leqslant(\Gamma(\alpha))^{-1} \int_{0}^{\delta} t^{-\left(\frac{1}{2} \nu+3 / 4\right)} d t \int_{0}^{t}(t-u)^{\frac{1}{2} \alpha-1}|\phi(u)| d u \\
&=(\Gamma(\alpha))^{-1} \int_{0}^{\delta}|\phi(u)| d u \int_{u}^{\delta}(t-u)^{\frac{1}{2} \alpha-1} t^{-\left(\frac{1}{2} \nu+3 / 4\right)} d t \\
&\left.\leqslant(\Gamma(\alpha))^{-1} \int_{0}^{\delta} u^{-\left(\frac{1}{2} \nu+3 / 4\right)} \mid \phi u\right) \mid d u \int_{u}^{\delta}(t-u)^{\frac{1}{2} \alpha-1} d t \\
&=\left(\frac{1}{2} \Gamma(\alpha+1)\right)^{-1} \int_{0}^{\delta} u^{-\left(\frac{1}{2} \nu+3 / 4\right)}(\delta-u)^{\frac{1}{2} \alpha}|\phi(u)| d u \\
& \leqslant 2 \delta^{\frac{1}{2} \alpha}(\Gamma(\alpha+1))^{-1} \int_{0}^{\delta} u^{-\left(\frac{1}{2} \nu+3 / 4\right)}|\phi(u)| d u<\infty .
\end{aligned}
$$

Hence

$$
{ }^{\nu+\alpha} L_{k, t}\left[s^{-\alpha} f(s)\right]
$$

exists for all $t>0$ and all $k>\gamma t$.

Now, for $b>\gamma t>0$,

$$
b^{-\alpha} f\left(\frac{b}{t}\right)=t^{1-\alpha} I_{\alpha}\left\{u^{-(\alpha+1)} f_{-\alpha}\left(\frac{b}{u}\right) ; t\right\} .
$$


For

$$
\begin{aligned}
t^{1-\alpha} I_{\alpha} & \left\{u^{-(\alpha+1)} f_{-\alpha}\left(\frac{b}{u}\right) ; t\right\} \\
& =\frac{t^{1-\alpha}}{\Gamma(\alpha)} \int_{0}^{t}(t-u)^{\alpha-1} u^{-(\alpha+1)} d u \int_{0}^{\infty} e^{-b v / u} v^{\alpha} \phi(v) d v \\
& =\frac{t^{1-\alpha}}{\Gamma(\alpha)} \int_{0}^{\infty} v^{\alpha} \phi(v) d v \int_{0}^{t} e^{-b v / u}(t-u)^{\alpha-1} u^{-(\alpha+1)} d u \\
& \left.=\frac{t^{-\alpha}}{\Gamma(\alpha)} \int_{0}^{\infty} e^{-b v / t} v^{\alpha} \phi(v) d v \int_{0}^{\infty} e^{-b v x / t} x^{\alpha-1} d x \quad \text { (where } x=(t / u)-1\right) \\
& =b^{-\alpha} \int_{0}^{\infty} e^{-b v / t} \phi(v) d v=b^{-\alpha} f\left(\frac{b}{v}\right) .
\end{aligned}
$$

The interchange of integrations is justified by Fubini's theorem, since $b>\gamma t$, and since, with the same change of variables as above,

$$
\frac{t^{1-\alpha}}{\Gamma(\alpha)} \int_{0}^{\infty} v^{\alpha}|\phi(v)| d v \int_{0}^{t} e^{-b v / u}(t-u)^{\alpha-1} u^{-(\alpha+1)} d u=b^{-\alpha} \int_{0}^{\infty} e^{-b v / t}|\phi(v)| d v<\infty,
$$

Thus, setting $b=k(x+1)$, we have

$$
\begin{aligned}
{ }^{\nu+\alpha} L_{k, t}[ & \left.s^{-\alpha} f(s)\right]=\frac{k^{3 / 2} e^{2 k}}{t \pi^{\frac{1}{2}}} \int_{0}^{\infty} x^{\frac{1}{2}(\nu+\alpha)} J_{\nu+\alpha}\left(2 k x^{\frac{1}{2}}\right)\left(\frac{k(x+1)}{t}\right)^{-\alpha} f\left(\frac{k(x+1)}{t}\right) d x \\
& =\frac{k^{3 / 2} e^{2 k}}{\pi^{\frac{1}{3}}} \int_{0}^{\infty} x^{\frac{1}{2}(\nu+\alpha)} J_{\nu+\alpha}\left(2 k x^{\frac{1}{2}}\right) I_{\alpha}\left\{u^{-(\alpha+1)} f_{-\alpha}\left(\frac{k(x+1)}{u}\right) ; t\right\} d x \\
& =I_{\alpha}\left\{\frac{u^{-\alpha} k^{3 / 2} e^{2 k}}{u \pi^{\frac{1}{2}}} \int_{0}^{\infty} x^{\frac{1}{2}(\nu+\alpha)} J_{\nu+\alpha}\left(2 k x^{\frac{1}{2}}\right) f_{-\alpha}\left(\frac{k(x+1)}{u}\right) d x ; t\right\} \\
& =\iota_{\alpha}\left\{u^{-\alpha(\nu+\alpha)} L_{k, u}\left[f_{-\alpha}(s)\right] ; t\right\} .
\end{aligned}
$$

The interchange of integrations is justified by Fubini's theorem. For, $k>\gamma t>0$, and the same argument used to show the existence of ${ }^{\nu+\alpha} L_{k, t}\left[s^{-\alpha} f(s)\right]$ can be applied to show the existence of ${ }^{\nu+\alpha} L_{k, t}\left[s^{-\alpha} \bar{f}(s)\right]$, and thus

$$
\begin{aligned}
& \frac{k^{3 / 2} e^{2 k}}{\pi^{\frac{1}{2}}} \int_{0}^{\infty} x^{\frac{1}{2}(\nu+\alpha)}\left|J_{\nu}\left(2 k x^{\frac{1}{2}}\right)\right| I_{\alpha}\left\{u^{-(\alpha+1)}\left|f_{-\alpha}\left(\frac{k(x+1)}{u}\right)\right| ; t\right\} d x \\
& \leqslant \frac{k^{3 / 2} e^{2 k}}{\pi^{\frac{1}{2}}} \int_{0}^{\infty} x^{\frac{1}{2}(\nu+\alpha)}\left|J_{\nu}\left(2 k x^{\frac{1}{2}}\right)\right| I_{\alpha}\left\{u^{-(\alpha+1)} \bar{f}_{-\alpha}\left(\frac{k(x+1)}{u}\right) ; t\right\} d x \\
& \quad=\frac{k^{3 / 2} e^{2 k}}{\pi^{\frac{1}{2}}} \int_{0}^{\infty} x^{\frac{1}{2}(\nu+\alpha)}\left|J_{\nu}\left(2 k x^{\frac{1}{2}}\right)\right|\left(\frac{k(x+1)}{t}\right)^{-\alpha} \bar{f}\left(\frac{k(x+1)}{t}\right) d x<\infty .
\end{aligned}
$$

But by (1), (2), and the corollary to Theorem 2.2,

$$
u^{-\alpha}{ }^{\nu+\alpha} L_{k, u}\left[f_{-\alpha}(s)\right]={ }^{\nu} L_{k, u}[f(s)] .
$$

Hence finally, for each $t>0$ and all $k>\gamma t$,

$$
{ }^{\nu+\alpha} L_{k, t}\left[s^{-\alpha} f(s)\right]=I_{\alpha}\left\{{ }^{\nu} L_{k, u}[f(s)] ; t\right\} .
$$

The following theorem gives sufficient conditions that ${ }^{\nu} L_{k, t}[f(s)]$ exist for a given $f(s)$, and yields a relation involving the Laplace transformation of the operator. 
THEOREM 2.4. If

(1) $e^{-\gamma u} u^{-1} f\left(u^{-1}\right) \in L(0, \infty), \gamma>0$,

(2) $u^{-\left(\frac{1}{2} v+7 / 4\right)} f\left(u^{-1}\right) \in L(0, \delta)$, for some $\delta>0$,

(3) $g(s)=\int_{0}^{\infty} e^{-s u} u^{-1} f\left(u^{-1}\right) d u, s>\gamma$,

(4) $\nu>-1$,

then

(i) ${ }^{\nu} L_{k, t}[f(s)]$ exists for each $k>0$ and almost all $t>0$,

$$
\sigma^{-1}{ }^{\nu} L_{k, \sigma^{-1}}[g(s)] \text { and } \int_{0}^{\infty} e^{-\sigma t{ }^{\nu}} L_{k, t}[f(s)] d t
$$

exist and are equal for each $\sigma>0$ and all $k>\gamma / \sigma$.

Proof. By Theorem 2.1,

$$
{ }^{\nu} L_{k, \sigma^{-1}}[g(s)] \text { and }{ }^{\nu} L_{k, \sigma^{-1}}[\bar{g}(s)]
$$

exist for $\sigma>0$ and all $k>\gamma / \sigma$. But,

$$
\begin{aligned}
\sigma^{-1 \nu} & L_{k, \sigma^{-1}}[g(s)]=\frac{k^{3 / 2} e^{2 k}}{\pi^{\frac{3}{3}}} \int_{0}^{\infty} x^{\frac{1}{2^{2} \nu}} J_{\nu}\left(2 k x^{\frac{1}{2}}\right) d x \int_{0}^{\infty} e^{-k(x+1) \sigma u} u^{-1} f\left(u^{-1}\right) d u \\
& \left.=\frac{k^{3 / 2} e^{2 k}}{\pi^{\frac{1}{2}}} \int_{0}^{\infty} x^{\frac{1}{z^{\nu} \nu}} J_{\nu}\left(2 k x^{\frac{1}{2}}\right) d x \int_{0}^{\infty} e^{-\sigma t} t^{-1} f\left(\frac{k(x+1)}{t}\right) d t \text { (where } t=k(x+1) u\right) \\
& =\frac{k^{3 / 2} e^{2 k}}{\pi^{3}} \int_{0}^{\infty} e^{-\sigma t} t^{-1} d t \int_{0}^{\infty} x^{\frac{1}{2} \nu} J_{\nu}\left(2 k x^{\frac{1}{2}}\right) f\left(\frac{k(x+1)}{t}\right) d x \\
& =\int_{0}^{\infty} e^{-\sigma t \nu} L_{k, t} d t .
\end{aligned}
$$

The interchange of integrations is justified by Fubini's theorem, since ${ }^{\nu} L_{k, \sigma^{-1}}[\bar{g}(s)]$ exists for $\sigma>0$ and all $k>\gamma / \sigma$, and hence

$$
\begin{gathered}
\int_{0}^{\infty} x^{\frac{1}{2} \nu}\left|J_{\nu}\left(2 k x^{\frac{1}{2}}\right)\right| d x \int_{0}^{\infty} e^{-k(x+1) \sigma u} u^{-1}\left|f\left(u^{-1}\right)\right| d u \\
\quad=\int_{0}^{\infty} x^{\frac{1}{\nu}}\left|J_{\nu}\left(2 k x^{\frac{1}{2}}\right)\right| \bar{g}(k(x+1) \sigma) d x<\infty .
\end{gathered}
$$

Thus, by Fubini's theorem ${ }^{\nu} L_{k, t}[f(s)]$ exists for each $k>0$ and almost all $t>0$, and has a Laplace transform, and the equality stated is true.

Corollary. If

(1) $e^{-\gamma u} u^{-1} f\left(u^{-1}\right) \in L(0, \infty), \gamma>0$,

(2) $g^{\epsilon}(s)=\int_{0}^{\infty} e^{-s u} u^{-1} e^{-\epsilon / u} f\left(u^{-1}\right) d u, s \geqslant \gamma, \epsilon>0$,

(3) $\nu>-1$,

\section{then}


(i) ${ }^{\nu} L_{k, t}\left[e^{-\epsilon s} f(s)\right]$ exists for each $k>0$ and almost all $t>0$,

$$
\sigma^{-1 \nu} L_{k, \sigma^{-1}}\left[g^{\epsilon}(s)\right] \text { and } \int_{0}^{\infty} e^{-\sigma t \nu} L_{k, t}\left[e^{-\epsilon s} f(s)\right] d t
$$

exist and are equal for each $\sigma>0$ and all $k>\gamma / \sigma$.

Proof. The hypotheses of Theorem 2.4 are clearly fulfilled relative to $e^{-\epsilon s} f(s)$ except possibly (2). But $u^{-\left(\frac{1}{2} v+3 / 4\right)} e^{-\epsilon / u}$ is bounded for $u>0$, and (2) is fulfilled also. Hence, applying the results of Theorem 2.4 to $e^{-\epsilon s} f(s)$, we arrive at the above conclusions.

The next theorem concerns the behaviour of ${ }^{\nu} L_{k, t}\left[e^{-\epsilon s} f(s)\right]$ when $\phi(t) \in$ $L_{p}(0, \infty), 1<p \leqslant \infty$.

Theorem 2.5. If $\phi(t) \in L_{p}(0, \infty)$, $p$ fixed, $1<p \leqslant \infty$, and $\nu>-1$, then

(i) ${ }^{\nu} L_{k, t}\left[e^{-\epsilon s} f(s)\right]$ exists if $k, t$, and $\epsilon$ are positive,

(ii) $k_{0}$ and $N$ exist, $N$ independent of $k$, such that for $k>k_{0}$,

$$
\left\|{ }^{\nu} L_{k, t}\left[e^{-\epsilon s} f(s)\right]\right\|_{p} \leqslant N\|\phi(t)\|_{p} .
$$

Proof. Existence follows from the corollary to Theorem 2.1, and for all positive $k$ since any positive $\gamma$ may be used.

Now ${ }^{\nu} L_{k, t}\left[e^{-\epsilon s} f(s)\right]$

$$
\begin{aligned}
& =\frac{k^{3 / 2} e^{2 k}}{t \pi^{\frac{3}{3}}} \int_{0}^{\infty} x^{\frac{1}{2} \nu} J_{\nu}\left(2 k x^{\frac{1}{2}}\right) e^{-\epsilon k(x+1) / t} d x \int_{0}^{\infty} e^{-k(x+1) u / t} \phi(u) d u \\
& =\frac{k^{3 / 2} e^{2 k}}{t \pi^{3}} \int_{0}^{\infty} e^{-k(u+\epsilon) / t} \phi(u) d u \int_{0}^{\infty} e^{-k(u+\epsilon) x / t} x^{\frac{1}{2} \nu} J_{\nu}\left(2 k x^{\frac{1}{2}}\right) d x \\
& =\frac{t^{\nu+1} k^{\frac{1}{2}} e^{2 k}}{t \pi^{3}} \int_{0}^{\infty} e^{-k\left((u+\epsilon) t^{-1}+(u+\epsilon)^{-1} t\right)}(u+\epsilon)^{-(\nu+1)} \phi(u) d u,
\end{aligned}
$$

where we have used $(3 ; \S 13.3,(3))$, and the interchange of integrations is justified by Fubini's theorem if $k>0, t>0$, since ${ }^{\nu} L_{k, t}\left[e^{-\epsilon s \bar{f}(s)]}\right.$ exists by the Corollary to Theorem 2.1 applied to $|\phi(t)|$, and thus,

$$
\int_{0}^{\infty} x^{\frac{1}{\nu} \nu}\left|J_{\nu}\left(2 k x^{\frac{1}{2}}\right)\right| e^{-\epsilon k(x+1) / t} d x \int_{0}^{\infty} e^{-k(x+1) u / t}|\phi(u)| d u<\infty .
$$

Hence, for $1<p<\infty$, we have, using Hölder's inequality,

$$
\begin{aligned}
& \left.\left.\right|^{\nu} L_{k, t}\left[e^{-\epsilon s} f(s)\right]\right|^{p} \\
& \leqslant\left(\frac{t^{\nu} k^{\frac{1}{2}} e^{2 k}}{\pi^{\frac{1}{3}}}\right)^{p} \int_{0}^{\infty} e^{\left.-k(u+\epsilon) t^{-1}+(u+\epsilon)^{-1} t\right)}(u+\epsilon)^{-(\nu+1)}|\phi(u)|^{p} d u \\
& \quad\left\{\int_{0}^{\infty} e^{-k\left(u t^{-1}-t u^{-1}\right)} u^{-(\nu+1)} d u\right\}^{p / q} \\
& \leqslant\left\{\frac{2 k^{\frac{1}{2}} e^{k} K_{\nu}(2 k)}{\pi^{\frac{3}{t}}}\right\}^{p / q} \frac{t^{\nu} k^{\frac{1}{3}} e^{2 k}}{\pi^{\frac{2}{3}}} \int_{0}^{\infty} e^{-k\left((u+\epsilon) t^{-1}+(u+\epsilon)^{-1} t\right)}(u+\epsilon)^{-(\nu+1)}|\phi(u)|^{p} d u .
\end{aligned}
$$

by $(3 ; \S 6.23,(8))$, where $q=p /(p-1)$. Hence 


$$
\begin{aligned}
& \int_{0}^{\infty}\left|L_{k, t}\left[e^{-\epsilon s} f(s)\right]\right|^{p} d t \\
& \leqslant\left\{\frac{2 k^{\frac{1}{2}} e^{2 k} K_{\nu}(2 k)}{\pi^{3}}\right\}^{p / q} \frac{k^{\frac{1}{2}} e^{2 k}}{\pi^{\frac{1}{2}}} \int_{0}^{\infty}|\phi(u)|^{p} d u \int_{0}^{\infty} e^{-k(u+\epsilon) t^{-1}+(u+\epsilon)-1} t t^{\nu}(u+\epsilon)^{-(\nu+1)} d t \\
& =\left\{\frac{2 k^{\frac{1}{2}} e^{2 k} K_{\nu}(2 k)}{\pi^{3}}\right\}^{p / q} \frac{2 k^{\frac{1}{2}} e^{2 k} K_{\nu+1}(2 k)}{\pi^{\frac{1}{3}}} \int_{0}^{\infty}|\phi(u)|^{p} d u .
\end{aligned}
$$

Hence

$$
\left\|{ }^{\nu} L_{k, t}\left[e^{-\epsilon s} f(s)\right]\right\|_{p} \leqslant\left\{\frac{2 k^{\frac{1}{2}} e^{2 k} K_{p}(2 k)}{\pi^{3}}\right\}^{1 / q}\left\{\frac{2 k^{\frac{1}{2}} e^{2 k} K_{\nu+1}(2 k)}{\pi^{\frac{1}{3}}}\right\}^{1 / p}\|\phi(t)\|_{p}
$$

But by $(3 ; \S 7.23,(1)), K_{\lambda}(2 k) \sim e^{-2 k} \pi^{\frac{1}{2}} / 2 k^{\frac{1}{2}}$ as $k \rightarrow \infty$. Hence $k_{0}(\lambda)$ and $N$ exist such that for $k>k_{0}, 2 k^{\frac{1}{2}} e^{2 k} K_{\lambda}(2 k) \pi^{-\frac{1}{2}} \leqslant N$. Thus if $k_{0} \geqslant \max \left(k_{0}(\nu)\right.$, $\left.k_{0}(\nu+1)\right)$, we have

$$
\left\|{ }^{\nu} L_{k, t}\left[e^{-\epsilon s} f_{(s)}\right]\right\|_{p} \leqslant N\|\phi(t)\|_{p}, \quad k>k_{0} .
$$

If $p=\infty$, we have

$$
\begin{aligned}
\left|{ }^{\nu} L_{k, t}\left[e^{-\epsilon s} f(s)\right]\right| \leqslant \frac{k^{\frac{1}{2}} e^{2 k}}{\pi^{3}} \int_{0}^{\infty} e^{-k\left(u t^{-1}+t u^{-1}\right)} t^{\nu} u^{-(v+1)} d u\|\phi(t)\|_{\infty} \\
=\frac{2 k^{\frac{1}{3}} e^{2 k} K_{\nu}(2 k)}{\pi^{3}}\|\phi(t)\|_{\infty} \leqslant N\|\phi(t)\|_{\infty},
\end{aligned}
$$

for $k>k_{0}(\nu)$, so that

$$
\left\|{ }^{\nu} L_{k, t}\left[e^{-\epsilon s} f(s)\right]\right\|_{\infty} \leqslant N\|\phi(t)\|_{\infty} .
$$

Corollary. If $\phi(t) \in L_{p}(0, \infty), p$ fixed, $1<p \leqslant \infty, p>4 /(1-2 \nu)$, and $\nu>-1$, then

(1) ${ }^{\nu} L_{k, t}[f(s)]$ exists if $k$ and $t$ are positive,

(2) $k_{0}$ and $N$ exist, $N$ independent of $k$, such that for $k>k_{0}$,

$$
\left\|{ }^{\nu} L_{k, t}[f(s)]\right\|_{p} \leqslant N\|\phi(t)\|_{p} .
$$

Proof. If $p>4 /(1-2 \nu)$, then $q\left(\frac{1}{2} \nu+3 / 4\right)<1$, where $q=p /(p-1)$. Hence, by Hölder's inequality,

$$
\int_{0}^{\delta} t^{-\left(\frac{3}{2} \nu+3 / 4\right)}|\phi(t)| d t \leqslant\left\{\int_{0}^{\delta} t^{-\alpha\left(\frac{1}{2} \nu+3 / 4\right)} d t\right\}^{1 / \alpha}\left\{\int_{0}^{\delta}|\phi(t)|^{p} d t\right\}^{1 / p}<\infty,
$$

so that, by Theorem $2.1,{ }^{\nu} L_{k, t}[f(s)]$ exists for $t>0$, and for all $k>0$ since any $\gamma>0$ may be used.

The remainder of the corollary follows in exactly the same manner as in Theorem 2.5.

3. Inversion of the transformation. The following three theorems comprise the inversion theory for the operator.

THEorem 3.1. If

(1) $e^{-\gamma^{t} \phi(t)} \in L(0, \infty), \quad \gamma>0$, and if

(2) $\nu \geqslant-\frac{1}{2}$ and $t^{-(\nu+1)} \phi(t) \in L(0, \delta)$ for some $\delta>0$, or if 
$\left(2^{\prime}\right)-1<\nu \leqslant \frac{1}{2}$ and $t^{-\left(\frac{1}{2} v+3 / 4\right)} \phi(t) \in L(0, \delta)$ for some $\delta>0$, then, at each point $t>0$ of the Lebesgue set of $\phi$,

$$
\lim _{k \rightarrow \infty}{ }^{\nu} L_{k, t}[f(s)]=\phi(t) .
$$

Proof. Case (a) $: \nu \geqslant-\frac{1}{2}$. Setting $\lambda=-\frac{1}{2}$ and $\mu=\nu+\frac{1}{2}$ in Theorem 2.2, we have that ${ }^{\nu} L_{k, t}[f(s)]$ and $t^{\left(v+\frac{1}{2}\right)-\frac{1}{2}} L_{k, t}\left[f_{v+\frac{1}{2}}(s)\right]$ exist and are equal. But by (2; Theorem 1$)$, under the above conditions

$$
\lim _{k \rightarrow \infty}{ }^{-\frac{1}{2}} L_{k, t}\left[f_{v+\frac{1}{2}}(s) \mid=t^{-\left(v+\frac{1}{2}\right)} \phi(t)\right.
$$

at each point $t>0$ of the Lebesgue set of $\phi$. Hence, at each such point,

$$
\lim _{k \rightarrow \infty}{ }^{\nu} L_{k, t}[f(s)]=\phi(t) .
$$

Case (b): $-1<\nu \leqslant \frac{1}{2}$. Setting $\lambda=\nu, \mu=-\left(\nu+\frac{1}{2}\right)$ in the Corollary to Theorem 2.2, we have that ${ }^{-\frac{1}{2}} L_{k, t}\left[f_{v+\frac{1}{2}}(s)\right]$ and $t^{-\left(v+\frac{1}{2}\right)}{ }^{\nu} L_{k, t}[f(s)]$ are equal. Hence, as in case (a), using (2; Theorem 1 ) we have

$$
\lim _{k \rightarrow \infty}{ }^{\nu} L_{k, t}[f(s)]=\phi(t)
$$

at each point $t>0$ of the Lebesgue set of $\phi$.

THEOREM 3.2. If

(1) $e^{-\gamma^{t} \phi}(t) \in L(0, \infty), \gamma>0$,

(2) $\alpha>0, \nu>-1$,

and if

(3) $\alpha+\nu \geqslant-\frac{1}{2}$ and $t^{-(\nu+1)} \phi(t) \in L(0, \delta)$ for some $\delta>0$, or if

$\left(3^{\prime}\right) \alpha+\nu \leqslant-\frac{1}{2}$ and $t^{-\left(\frac{1}{2} \nu+3 / 4\right)} \phi(t) \in L(0, \delta)$ for some $\delta>0$, then for each $t>0$, of the Lebesgue set of $I_{\alpha}\{\phi(u) ; t\}$

$$
\lim _{k \rightarrow \infty} I_{\alpha}\left\{{ }^{\nu} L_{k, u}[f(s)] ; t\right\}=I_{\alpha}\{\phi(u) ; t\} .
$$

Proof. By Theorem 2.3, under the above conditions

$$
I_{\alpha}\left\{{ }^{\nu} L_{k, u}[f(s)] ; t\right\}={ }^{\nu+\alpha} L_{k, t}\left[s^{-\alpha} f(s)\right] .
$$

Thus the theorem will follow from Theorem 3.1, if either $\nu+\alpha \geqslant-\frac{1}{2}$ and $t^{-(\nu+\alpha+1)} I_{\alpha}\{\phi(u) ; t\} \in L(0, \delta)$ for some $\delta>0$, or $\nu+\alpha \leqslant-\frac{1}{2}$ and $t^{\left.-\frac{1}{2}(\nu+\alpha)+3 / 4\right)}$ $I_{\alpha}\{\phi(u) ; t\} \in L(0, \delta)$ for some $\delta>0$. But if $\nu+\alpha \geqslant-\frac{1}{2}$, then $t^{-(v+1)}$ $\phi(t) \in L(0, \delta)$, and thus

$$
\begin{aligned}
& \int_{0}^{\delta} t^{-(\nu+\alpha+1)}\left|I_{\alpha}\{\phi(u) ; t\}\right| d t \leqslant(\Gamma(\alpha))^{-1} \int_{0}^{\delta} t^{-(v+\alpha+1)} d t \int_{0}^{t}(t-u)^{\alpha-1}|\phi(u)| d u \\
& \quad=(\Gamma(\alpha))^{-1} \int_{0}^{\delta}|\phi(u)| d u \int_{u}^{\delta} t^{-(\nu+\alpha+1)}(t-u)^{\alpha-1} d t \\
& \left.\quad=(\Gamma(\alpha))^{-1} \int_{0}^{\delta} u^{-(v+1)}|\phi(u)| d u \int_{u / \delta}^{1} v^{\nu}(1-v)^{\alpha-1} d v \quad \quad \text { (where } v=u / t\right) \\
& \quad \leqslant(\Gamma(\alpha))^{-1} \int_{0}^{\delta} u^{-(\nu+1)}|\phi(u)| d u \int_{0}^{1} v^{\nu}(1-v)^{\alpha-1} d v<\infty .
\end{aligned}
$$


Further, we have already shown in the course of the proof of Theorem 2.3 that if $t^{-\left(\frac{1}{2} v+3 / 4\right)} \phi(t) \in L(0, \delta)$, then so is $t^{-\left(\frac{1}{2}(\nu+\alpha)+3 / 4\right)} I_{\alpha}\{\phi(u) ; t\}$.

We conclude this section with a theorem removing the restrictions on the behaviour of $\phi(t)$ at $t=0$.

THEOREM 3.3. If

(1) $e^{-\gamma t} \phi(t) \in L(0, \infty), \gamma>0$,

(2) $\nu>-1$.

then for each $\epsilon>0$, and at each point $t>\epsilon$ such that $t-\epsilon$ is in the Lebesgue set of $\phi(u)$, we have

$$
\lim _{k \rightarrow \infty}{ }^{\nu} L_{k, t}\left[e^{-\epsilon s} f(s)\right]=\phi(t-\epsilon) .
$$

Proof. We have $e^{-\epsilon s} f(s)=\int_{0}^{\infty} e^{-s t} \phi_{\epsilon}(t) \mathrm{dt}$, where $\phi_{\epsilon}(t)=0,0<t<\epsilon$, and $\phi_{\epsilon}(t)=\phi(t-\epsilon), t>\epsilon$. Thus the hypotheses of Theorem 3.1 are clearly fulfilled relative to $\phi_{\epsilon}(t)$, and the conclusion follows.

4. Representation theorems. The first theorem of this section is fundamental in the representation theory.

THEOREM 4.1. If

(1) $e^{-\gamma u} u^{-1} f\left(u^{-1}\right) \in L(0, \infty), \gamma>0$,

and if

(2) $\nu \geqslant-\frac{1}{2}$, and $u^{-(\nu+2)} f\left(u^{-1}\right) \in L(0, \delta)$ for some $\delta>0$, or if

$\left(2^{\prime}\right)-1<\nu \leqslant-\frac{1}{2}$, and $u^{-\left(\frac{1}{2} \nu+7 / 4\right)} f\left(u^{-1}\right) \in L(0, \delta)$ for some $\delta>0$,

then ${ }^{\nu} L_{k, t}[f(s)]$ exists for each $k>0$ and almost all $t>0,{ }^{\nu} L_{k, t}[f(s)]$ has a Laplace transform, and

$$
\lim _{k \rightarrow \infty} \int_{0}^{\infty} e^{-\sigma t \nu} L_{k, t}[f(s)] d t=f(\sigma)
$$

at each point $\sigma>0$ of the Lebesgue set of $f$.

Proof. The existence of ${ }^{\nu} L_{k, t}[f(s)]$ follows from Theorem 2.4, as does the existence of its Laplace transform for each $\sigma>0$ and all $k>\gamma / \sigma$. The remainder of the theorem follows from Theorems 2.4 and 3.1.

COROLlary. If

(1) $e^{-\gamma u} u^{-1} f\left(u^{-1}\right) \in L(0, \infty), \gamma>0$,

(2) $\nu>-1, \epsilon>0$,

then ${ }^{\nu} L_{k, t}\left[e^{-\epsilon s} f(s)\right]$ exists for each $k>0$ and almost all $t>0,{ }^{\nu} L_{k, t}\left[e^{-\epsilon s} f(s)\right]$ has a Laplace transform, and

$$
\lim _{k \rightarrow \infty} \int_{0}^{\infty} e^{-\sigma t \nu} L_{k, t}\left[e^{-\epsilon s} f(s)\right] d t=e^{-\epsilon \sigma} f(\sigma),
$$

at each point $\sigma>0$ of the Lebesgue set of $f$.

Proof. Since $u^{-(\nu+1)} e^{-\epsilon / u}$ and $u^{-\left(\frac{1}{2} v+7 / 4\right)} e^{-\epsilon / u}$ are uniformly bounded in $u$ for $u>0$, the hypotheses of Theorem 4.1 are satisfied, and the conclusion follows. 
THEOREM 4.2. Necessary and sufficient conditions that $f(s)$ be equal almost everywhere to the Laplace transform of a function of the form $t^{\alpha} \phi(t)$, where $\phi(t) \in L_{p}(0, \infty), p$ fixed, $1<p \leqslant \infty$ and $\alpha>-(p-1) / p$, are that

(1) $e^{-\gamma u} u^{-1} f\left(u^{-1}\right) \in L(0, \infty), \gamma>0$,

(2) $\left\|t^{-\alpha \nu+\alpha} L_{k, t}\left[e^{-\epsilon} s f(s)\right]\right\|_{p} \leqslant M_{p}$,

where $M_{p}$ is independent of $\epsilon$ and $k$, for all $\epsilon>0$ and $k>k_{0}$, for some $\nu>\max (-1,-1-\alpha)$.

Proof of necessity. Let

$$
f(s)=\int_{0}^{\infty} e^{-s t} t^{\alpha} \phi(t) d t,
$$

where $\phi(t) \in L_{p}(0, \infty)$, $p$ fixed, $1<p \leqslant \infty$, and $\alpha>-(p-1) / p$.

If $p<\infty$, and $q=p /(p-1)$, we have by Hölder's inequality, for $s>0$,

$$
\begin{aligned}
|f(s)| & \leqslant\left\{\int_{0}^{\infty}|\phi(t)|^{p} d t\right\}^{1 / p}\left\{\int_{0}^{\infty} e^{-q s t} t^{\alpha q} d t\right\}^{1 / q} \\
& =(q s)^{-(\alpha+1 / q)}(\Gamma(1+\alpha q))^{1 / q}\|\phi(t)\|_{p}=M s^{-(\alpha+1 / q)} .
\end{aligned}
$$

Thus, for any $\gamma>0$,

$$
e^{-\gamma u} u^{-1}\left|f\left(u^{-1}\right)\right| \leqslant M e^{\gamma u-} u^{-(1-a-1 / q)}=M e^{-\gamma u} u^{a-1 / p}
$$

and hence since $\alpha-1 / p>-p / p=-1$, we find that $e^{-\gamma u} u^{-1} f\left(u^{-1}\right) \in L(0, \infty)$.

If $p=\infty$, we have for $s>0$,

$$
|f(s)| \leqslant \int_{0}^{\infty} e^{-s t} t^{\alpha} d t\|\phi(t)\|_{\infty}=s^{-(\alpha+1)} \Gamma(\alpha+1)\|\phi(t)\|_{\infty},
$$

and hence for any $\gamma>0$, since $\alpha>-1$,

$$
e^{-\gamma u} u^{-1}\left|f\left(u^{-1}\right)\right| \leqslant e^{-\gamma u} u^{\alpha} \Gamma(\alpha+1)\|\phi(t)\|_{\infty} \in L(0, \infty) .
$$

For the necessity of (2), we have by Theorem 2.2, if $\alpha \geqslant 0$,

where

$$
t^{-\alpha \nu+\alpha} L_{k, t}\left[e^{-\epsilon s} f(s)\right]={ }^{\nu} L_{k, t}\left[\left(e^{-\epsilon s} f(s)\right)_{\alpha}\right]={ }^{\nu} L_{k, t}\left[e^{-\epsilon s} g(s)\right]
$$

$$
g(s)=\int_{0}^{\infty} e^{-s t} t^{\alpha}(t+\epsilon)^{-\alpha} \phi(t) d t
$$

If $\alpha<0$, we set $\lambda=\nu+\alpha, \mu=-\alpha$ in the corollary to Theorem 2.2 and obtain again

But

$$
t^{-\alpha \nu+\alpha} L_{k, t}\left[e^{-\epsilon} f(s)\right]={ }^{\nu} L_{k, t}\left[e^{-\epsilon s} g(s)\right]
$$

$$
g(s)=\int_{0}^{\infty} e^{-s t} t^{\alpha}(t+\epsilon)^{-\alpha} \phi(t) d t
$$

is the Laplace transform of a function in $L_{p}$. In fact, since $t^{\alpha}(t+\epsilon)^{-\alpha}<1$ for $t \geqslant 0$, we have $\left\|t^{\alpha}(t+\epsilon)^{-\alpha} \phi(t)\right\|_{p} \leqslant\|\phi(t)\|_{p}<\infty$. Hence we may apply Theorem 2.5, and obtain, for $k>k_{0}$,

$\left\|t^{-\alpha \nu+\alpha} L_{k, t}\left[e^{-\epsilon s} f(s)\right]\right\|_{p}=\left\|{ }^{\nu} L_{k, t}\left[e^{-\epsilon s} g(s)\right]\right\|_{p} \leqslant N\left\|t^{\alpha}(t+\epsilon)^{-\alpha} \phi(t)\right\|_{p} \leqslant N\|\phi(t)\|_{p}$. 
Proof of sufficiency. By (2) and (4; ch. 1, Theorem 17a for $1<p<\infty$, Theorem $17 \mathrm{~b}$ for $p=\infty$ ), there exists, for each $\epsilon>0$, an increasing unbounded sequence $\left\{k_{i}(\epsilon)\right\}$, and a function $\phi_{\epsilon}(t) \in L_{p}(0, \infty)$, with $\left\|\phi_{\epsilon}(t)\right\| \leqslant M_{p}$, such that

$$
\lim _{i \rightarrow \infty} \int_{0}^{\infty} \alpha(t) t^{-\alpha \nu+\alpha} L_{k_{i}, t}\left[e^{-\epsilon s} f(s)\right] d t=\int_{0}^{\infty} \alpha(t) \phi_{\epsilon}(t) d t
$$

for every function $\alpha(t) \in L_{q}(0, \infty)$. But for each $\sigma>0, e^{-\sigma t} t^{\alpha} \in L_{q}(0, \infty)$, since $\alpha>-1 / q$. Hence, by the Corollary to Theorem 4.1, we have for almost all $\sigma>0$

$$
\begin{aligned}
e^{-\epsilon \sigma} f(\sigma) & =\lim _{i \rightarrow \infty} \int_{0}^{\infty} e^{-\sigma t \nu+\alpha} L_{k_{i}, t}\left[e^{-\epsilon s} f(s)\right] d t \\
& =\lim _{i \rightarrow \infty} \int_{0}^{\infty} e^{-\sigma t} t^{\alpha} t^{-\alpha \nu+\alpha} L_{k_{i}, t}\left[e^{-\epsilon s} f(s)\right] d t=\int_{0}^{\infty} e^{-\sigma t} t^{\alpha} \phi_{\epsilon}(t) d t .
\end{aligned}
$$

But by the same theorems of (4), and since $\left\|\phi_{\epsilon}(t)\right\|_{p} \leqslant M_{p}$, there exists a sequence $\left\{\epsilon_{i}\right\}$, with $\lim \epsilon_{i}=0$, and a function $\phi(t) \in L_{p}(0, \infty)$ such that $\|\phi(t)\| \leqslant M_{p}$, and such that for every $\alpha(t) \in L_{q}(0, \infty)$,

$$
\lim _{i \rightarrow \infty} \int_{0}^{\infty} \alpha(t) \phi_{\epsilon_{i}}(t) d t=\int_{0}^{\infty} \alpha(t) \phi(t) d t .
$$

Let $\Sigma_{\epsilon}$ be the set of measure zero in which, for $\sigma>0$,

let

$$
e^{-\epsilon \sigma} f(\sigma) \neq \int_{0}^{\infty} e^{-\sigma t} t^{\alpha} \phi_{\epsilon}(t) d t
$$

Then $\Sigma$ has measure zero.

$$
\Sigma=\bigcup_{i=1}^{\infty} \Sigma_{\epsilon_{i}}
$$

Let $\sigma$ be positive and $\sigma \notin \Sigma$. Then since $e^{-\sigma t} t^{\alpha} \in L_{q}(0, \infty)$, we have

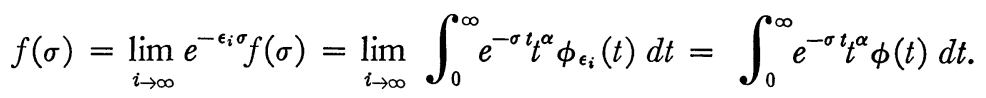

and the theorem is proved.

Corollary. Necessary and sufficient conditions that $f(s)$ be equal almost everywhere to the Laplace transform of a function in $L_{p}(0, \infty), p$ fixed, $1<p \leqslant \infty$, are that

(1) $e^{-\gamma u} u^{-1} f\left(u^{-1}\right) \in L(0, \infty), \gamma>0$,

(2) $\left\|{ }^{\nu} L_{k, t}\left[e^{-\epsilon s} f(s)\right]\right\|_{p} \leqslant M_{p}$, for all $\epsilon>0$ and $k>k_{0}$, and for some $\nu>-1$.

Proof. Since $(p-1) / p>0$, we may always set $\alpha=0$ in Theorem 4.2 and the result follows.

The next theorem shows how far the factor $e^{-\epsilon s}$ is necessary.

Theorem 4.3. Sufficient conditions that $f(s)$ be equal to the Laplace transformation of a function of the form $t^{\alpha} \phi(t), \phi(t) \in L_{p}(0, \infty)$, p fixed, $1<p \leqslant \infty$, $\alpha>-(p-1) / p$, are that 
(1) $e^{-\gamma u} u^{-1} f\left(u^{-1}\right) \in L(0, \infty), \gamma>0$, and

(2) $u^{-(\nu+\alpha+2)} f\left(u^{-1}\right) \in L(0, \delta)$ for some $\delta>0$, and some $\nu>-1$ such that $\nu+\alpha \geqslant-\frac{1}{2}$,

or

$\left(2^{\prime}\right) u^{-\left(\frac{1}{2}(\nu+\alpha)+7 / 4\right)} f\left(u^{-1}\right) \in L(0, \delta)$ for some $\delta>0$, and some $\nu$ such that $\nu>-1$, and $-1<\nu+\alpha \leqslant-\frac{1}{2}$,

(3) $\left\|t^{-\alpha \nu+\alpha} L_{k, t}[f(s)]\right\|_{p} \leqslant M_{p}, k>k_{0}$.

Condition (1) is necessary for all such $p$ and $\alpha$. Condition (2) is necessary if $p \nu>-1$, and $\left(2^{\prime}\right)$ is necessary if $p(1+2(\alpha-\nu))>4$. Condition (3) is necessary if $p>4 /(1-2 \nu)$.

Proof of necessity. Let

$$
f(s)=\int_{0}^{\infty} e^{-s t} t^{\alpha} \phi(t) d t
$$

where $\phi(t) \in L_{p}(0, \infty)$, $p$ fixed, $1<p \leqslant \infty$, and $\alpha>-(p-1) / p$.

The proof of the necessity of (1) is the same as in the preceding theorem, and, as in its proof, we have $|f(s)| \leqslant M s^{-(\alpha+1 / 4)}$ where $q=p /(p-1)$.

Hence

$$
u^{-(\nu+\alpha+2)}\left|f\left(u^{-1}\right)\right| \leqslant M u^{-(\nu+1+1 / p)},
$$

and thus (2) is necessary if $\nu+1 / p>0$, i.e., if $p \nu>-1$. Also,

$$
u^{-\left(\frac{1}{2}(\nu+\alpha)+7 / 4\right)}\left|f\left(u^{-1}\right)\right| \leqslant M u^{-\left(\frac{1}{2}(\nu-\alpha)+1 / p+\frac{3}{4}\right)}
$$

so that $\left(2^{\prime}\right)$ is necessary if $\frac{1}{2}(\nu-\alpha)+1 / p+\frac{3}{4}<1$, i.e., if $p(1+2(\alpha-\nu))>4$.

For the necessity of $(3)$, we have, since $p>4 /(1-2 \nu)$ that $q\left(\frac{1}{2} \nu+\frac{3}{4}\right)<1$. Hence if $\alpha \geqslant 0$, the hypotheses of Theorem 2.2 are fulfilled, and $t^{-\alpha \nu+\alpha} L_{k, t}[f(s)]={ }^{\nu} L_{k, t}\left[f_{\alpha}(s)\right]$. Thus by the Corollary to Theorem 2.4, since $f_{\alpha}(s)$ is the Laplace transform of $\phi(t)$, and $\phi(t) \in L_{p}(0, \infty)$,

$$
\left\|t^{-\alpha \nu+\alpha} L_{k, t}[f(s)]\right\|_{p} \leqslant N\|\phi(t)\|_{p} .
$$

If $\alpha \leqslant 0$, and if we set $\lambda=\nu+\alpha, \mu=-\alpha$, the hypotheses of the Corollary to Theorem 2.2 are fulfilled and the same results ensue. Hence (3) is necessary.

Proof of sufficiency. By $(3)$ and $(4 ;$ ch. 1 , Theorem 17 a for $1<p<\infty$, Theorem $17 \mathrm{~b}$ for $p=\infty$ ) there exists an increasing unbounded sequence $\left\{k_{i}\right\}$ and a function $\phi(t) \in L_{p}(0, \infty)$, with $\|\phi(t)\|_{p} \leqslant M_{p}$, and such that

$$
\lim _{i \rightarrow \infty} \int_{0}^{\infty} \alpha(t) t^{-\alpha \nu+\alpha} L_{k_{i}, t}[f(s)] d t=\int_{0}^{\infty} \alpha(t) \phi(t) d t
$$

for every function $\alpha(t) \in L_{q}(0, \infty)$. But for each $\sigma>0, e^{-\sigma t} t^{\alpha} \in L_{q}(0, \infty)$ since $\alpha>-1 / q$. Thus, by Theorem 4.1, we have for almost all $\sigma>0$,

$$
\begin{aligned}
f(\sigma) & =\lim _{i \rightarrow \infty} \int_{0}^{\infty} e^{-\sigma t v} L_{k_{i}, t}[f(s)] d t \\
& =\lim _{i \rightarrow \infty} \int_{0}^{\infty} e^{-\sigma t} t^{\alpha}\left(t^{-\alpha \nu} L_{k_{i}, t}[f(s)]\right) d t \\
& =\int_{0}^{\infty} e^{-\sigma t} t^{\alpha} \phi(t) d t .
\end{aligned}
$$




\section{REFERENCES}

1. A. Erdélyi, The inversion of the Laplace integral, Math. Mag., 24 (1950-51), 1-7.

2. P. G. Rooney, $A$ new inversion and representation theory for the Laplace transformation, Can. J. Math., 4 (1953), 436-444.

3. G. N. Watson, A treatise on Bessel functions (2nd. ed., New York, 1941).

4. D. V. Widder, The Laplace Transform (Princeton, 1941).

University of Alberta, and

Summer Research Institute 